\title{
1 Phenotypic Plasticity as an Intrinsic Property of Organisms
}

\author{
Sonia E. Sultan \\ Wesleyan University
}

\section{CONTENTS}

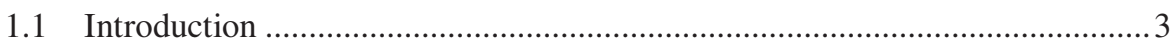

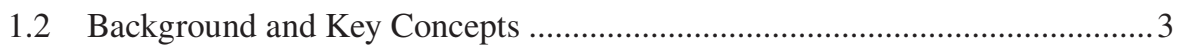

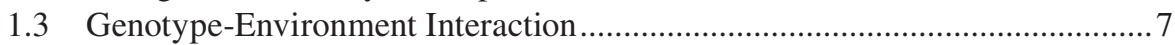

1.4 Studying Plasticity: Environmental Cues and Phenotypic Responses ........... 10

1.5 Transgenerational Plasticity and Multi-generation Norms of Reaction:

A More Complex Picture ........................................................................... 13

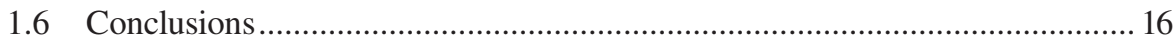

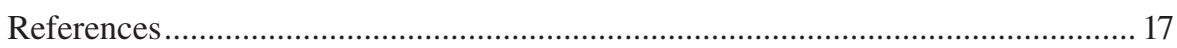

\subsection{INTRODUCTION}

It is a familiar biological observation that the traits of an organism will vary to some extent depending on environmental conditions. This individual flexibility is termed 'phenotypic plasticity,' which can be defined as the ability of a given genotype to express different phenotypes in different environmental circumstances. This opening chapter of the book provides some essential conceptual and practical tools to engage with this remarkable property of organisms. Starting with a background section that lays out the key observations and ideas, the chapter goes on to examine biological and statistical aspects of genotype-environment interaction; experimental approaches to studying plasticity; and the recently emerged area of transgenerational plasticity, including some new insights regarding multi-generational environmental influences.

\subsection{BACKGROUND AND KEY CONCEPTS}

Over the past four decades, plastic responses have been documented across the phylogenetic spectrum - in bacteria, fungi, and lichens; algae and land plants; marine and freshwater invertebrates; insects, fish, amphibians, reptiles, and mammals-in response to contrasting states of a broad range of abiotic and biotic factors including temperature and humidity; concentration of $\mathrm{O}_{2}$ and $\mathrm{CO}_{2}, \mathrm{pH}$, and other aspects of substrate and atmospheric chemistry; spectral quality, quantity, and diurnal pattern 
of light; type and availability of food and other resources; population density and social interactions; presence and density of competitors, predators, herbivores, pathogens, or mutualists; even (for both animals and plants) vibration, touch, and acoustic stimuli (references in Sultan 2000, 2007, 2015; Gilbert 2001; Gilbert and Epel 2009; 2015).

Plasticity encompasses all aspects of the phenotype in which expression varies as a result of environmental differences. Depending on the type of organism and the environmental factor, this can include such traits as growth rate and body mass; size, relative allocation, number, shape, or anatomical structure of parts and organs; metabolic rates and other physiological processes; composition and quantity of defensive compounds and other secondary products; behavior; developmental and life-history timing; sex expression; and reproductive output (op. cit.). Correlations among traits may also vary plastically in magnitude or direction (Gebhardt and Stearns 1993; Brock and Weinig 2007; Fischer et al. 2016). Because plasticity causes variation in functional and fitness traits and in traits that contribute to all kinds of species interactions, it is now recognized to be of fundamental importance to both ecology and evolution.

Broadly different types of organisms are characterized by distinctive modes of plastic expression. Microorganisms exhibit characteristic aspects of plasticity such as cellular movement and stress-based aggregation in response to microscale conditions including substrate texture and resource distribution (Seymour et al. 2010; Rivera-Yoshida et al. 2019). As modular organisms, plants express developmental as well as physiological plasticity throughout the life cycle, continuously adjusting to changing conditions at the level of cells and tissues, and altering the morphology, structure, and number of organs produced via multiple meristematic growing points that can respond independently to environmental signals (Gilroy and Trewavas 2001; Trewavas 2015). By contrast, with some exceptions (such as sponges and corals) most animals have fixed body plans with relatively canalized structural traits, and tissue plasticity is generally more limited to juvenile stages (Walbot 1996; Atkinson and Thorndyke 2001). As a result, during adulthood animals may express plasticity largely via behavioral and physiological adjustments (Dufty et al. 2002). However, differences between animals and plants should not be overstated (Huey et al. 2002); even reptiles, birds, and mammals can undergo rapid, reversible changes, plastically altering such adult traits as gut morphology, musculature, and organ size in response to seasonal change or new biotic challenges (Piersma and Lindström 1997; e.g. Van den Hout et al. 2006). And although plants lack the integrated multi-trait responses to their environments made possible by the animal brain and associated neuroendocrine system, confluences and crosstalk among hormonally mediated signaling pathways allow for a surprising degree of response coordination at the level of the whole plant body, just as in animals (Coupe et al. 2006; Hodge 2009).

Researchers have been particularly fascinated by the many cases in which plastic responses are appropriate to the conditions that elicit them, suggesting that plasticity can be adaptive (see Pfennig 2021 in this volume). Familiar examples include the production of broad, thin leaves by plants in low light, the enlarged feeding structures produced by certain invertebrate larvae in response to low food concentration, and the varied structural and chemical defenses induced by predator and herbivore 
attack. Yet plasticity is not always functionally adaptive. For instance, an animal or plant growing in resource-poor conditions will inevitably grow less and consequently produce fewer progeny. This would be considered maladaptive plasticity. At the same time, this resource-deprived individual may increase its relative biomass allocation to foraging organs or to reproduction, or adjust its rates of nutrient uptake or use, so as to partially compensate for these inevitable growth limits via adaptive aspects of plasticity. Phenotypes produced in response to stressful physical conditions may likewise reflect both inevitable limits and appropriate adjustments: in low temperature, for instance, developmental and metabolic rates will be unavoidably reduced, while plasticity for surface-to-volume ratio, thickness of insulating tissues, or body positioning may mediate the impact of low temperatures by maximizing heat conservation. Because phenotypic expression pathways are shaped by developmental and phylogenetic constraints as well as by natural selection, the phenotype an organism produces in a given environment will reflect both functionally adaptive and developmentally inevitable aspects of plastic response (Sultan and Stearns 2005).

Many authors have published definitions of plasticity, all of which are variations on a single key theme-phenotypic change in individual organisms that is associated with different environments. However, as with other fundamental biological concepts (for example, 'gene;' Fox Keller and Harel 2007; Portin and Wilkins 2017), there is a diversity of opinion about the precise definition of plasticity that reveals underlying tensions surrounding the term's meaning and scope. The definition given at the outset of this chapter - the ability of a given genotype to express different phenotypes in different environmental circumstances- has a long history and is quite widely used. Other definitions shift the ability to change from the genotype to the organism as a whole (e.g., Agrawal 2001). Both of these definitions emphasize that plasticity results from the genotype's or organism's response to its environment. A number of authors (such as Stearns 1989) instead define plastic phenotypic changes as induced by the environment, implying that the organism is more passive in this process. Although these alternatives suggest opposite directions of causation, they are in fact equally accurate, since plasticity results from the interaction of an organism with its environment (see next section). In her classic 2003 book, West-Eberhard provides a nuanced definition of plasticity that embraces this duality: "Conditionsensitive development or the ability of an organism to react to an environmental input with a change in form, state, movement, or rate of activity."

There is also variation in the way the term is applied. Certain authors may consider plasticity to be inherently adaptive (implicitly emphasizing the role of past selection in shaping plastic responses), though others (starting with Bradshaw's foundational 1965 paper) explicitly note that plasticity need not be adaptive, instead emphasizing developmental processes as such and their dependence on physical and chemical conditions. With respect to the scope of plasticity, the term may extend very broadly to encompass all aspects of phenotypic change that reflect an organism's experience (e.g., use and disuse of parts), or be more narrowly confined to "direct response to stimuli or inputs from the environment" (Pfennig 2021 in this volume). Most authors agree that plasticity "covers all types of environmentally induced phenotypic variation" (Stearns 1989). Those who instead use the term 'developmental plasticity' may do so in an equally inclusive way (e.g., West-Eberhard 2003) or may be (explicitly 
or implicitly) limiting their scope to structural features and excluding behavioral, biochemical, and metabolic responses (e.g., Piersma and Drent 2003).

Although plasticity is an obvious feature of living systems, environmentally flexible outcomes fit awkwardly into the simplified view of phenotypic causation that dominated both developmental and evolutionary biology in the mid-to-late 20th century (Sultan 1992; Sarkar 2004; and references therein). In this 'gene-for-trait' model, a specific DNA sequence was understood to directly determine a particular phenotypic outcome, and plasticity was considered an odd exception to this rule that posed a 'problem' for evolutionary research (Gilbert 2001; e.g., Stebbins 1980; Bonner 1988). More recently, sophisticated molecular studies have led to the key recognition that environmental conditions participate in gene regulatory pathways, both through direct inputs and indirectly via physiological changes to internal/intracellular states (Lewontin 2000; Nijhout 2003; Schlichting and Smith 2002; Gottlieb 2004; Carroll et al. 2005; Lemos et al. 2008; Gilbert 2012, Stinchcombe et al. 2012; and references therein), resulting in "an incredible degree of plasticity in gene expression in response to diverse environmental conditions" (Wray et al. 2014). The fundamental insight that gene expression is environmentally sensitive provides our starting point: plasticity is an intrinsic property of organisms.

With this principle in mind, the central approach to studying plasticity is to characterize each genotype's 'norm of reaction': the set of phenotypes it produces across a given range of environments, for any trait of interest (Figure 1.1; for a historical overview, see Sarkar 2004). The norm of reaction documents a genotype's repertoire of potential outcomes; it specifies that every phenotype results from the genotype's expression in response to a particular environment.

Note that a genotype's responses may be either constant across a specified environmental range ('canalized'), or markedly different from one environmental state to another (whether continuously 'plastic' or discretely 'polyphenic'; Figure 1.1). It is

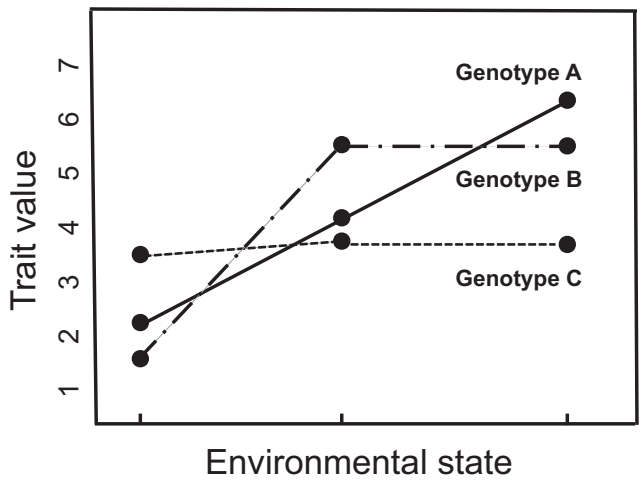

FIGURE 1.1 The norm of reaction. For every genotype, the phenotype for a trait of interest that is expressed in each specified environmental state is measured, and the points are joined to form a response curve (reaction norm). The reaction norm for a given trait may vary continuously (genotype A), consist of a few discrete alternatives (genotype B, polyphenism), or be relatively flat or canalized across the set of test environments (genotype C). (Modified from Sultan 2015.) 
important to understand that these alternative patterns do not reflect genetic versus environmental control of the trait. In other words, 'plastic' and 'canalized' are terms that describe different patterns of variation across a certain range of environmentsdifferent norms of reaction-but not different biological causes (Sultan 2015). Whether the phenotypes produced by a genotype in alternative treatments are similar or different, each phenotype results from that genome's expression under particular conditions. Note too that a genotype cannot be characterized as entirely 'plastic' versus 'canalized.' For a given trait, the same genotype may have a plastic response to one set of environmental states but a canalized response to other factors or factor levels, and it will express plasticity in some traits but not others. In general, then, norms of reaction for a given species are specific to the genotype, the set of environments, and the trait in question.

Experimentally, plastic responses are determined by generating replicate individuals of each genotype via cloning or inbreeding, and raising the isogenic replicates in two or more different environments of interest, such as contrasting temperature, $\mathrm{pH}$, or resource levels, or the presence versus absence of a biotic signal (such as a kairomone or plant volatile) or interactant (e.g., a competitor or predator; less precise experiments may instead use split sibships or genetically mixed samples from a given population or species as test groups instead of isogenic replicates; Sultan and Stearns 2005). This experimental strategy for characterizing genotypic reaction norms goes back over 75 years (e.g., Dobzhansky and Spassky 1944; Gupta and Lewontin 1982; for even earlier studies, see references in Sarkar 2004). The recently emerged discipline of 'ecological developmental biology' (or 'eco-devo') builds upon and expands this approach to include the underlying mechanisms as well as the outcomes of phenotypic expression in environmental context (Gilbert 2001; Sultan 2007; Gilbert and Epel 2009, 2015). Eco-devo studies examine how different possible environmental conditions may alter the signaling pathways that underlie development, physiology, behavior, and life-history. An eco-devo approach thus contrasts with the conventional study of development in a single-and often highly artificial-'control' environment (Gilbert 2001). Just like earlier norm of reaction experiments, eco-devo studies explicitly consider how genetic and environmental factors jointly determine phenotypes, starting at the level of gene expression. This recognition makes it clear that there is no neutral 'control' environment; an organism always develops in response to a specific set of conditions, even if those conditions consist of a petri plate on a lab bench.

\subsection{GENOTYPE-ENVIRONMENT INTERACTION}

These insights have crucial implications for understanding genetic diversity as well as development, and hence are of central relevance to evolutionary biology. Because DNA sequence differences affect signaling pathways for environmental perception and transduction as well as resulting impacts at the molecular and cellular levels, different genotypes in a given species will express characteristic norms of reaction; these distinct norms are rarely parallel to each other, since (as noted in Falconer's foundational Introduction to Quantitative Genetics) certain genotypes are "more sensitive than others to environmental differences" (Falconer 1989, p. 135; see also 
Haldane 1946; Gillespie and Turelli 1989; Kruuk et al. 2008; Mackay et al. 2009; Moczek et al. 2011; Des Marais et al. 2013). In quantitative genetics, non-parallel reaction norms are described by the statistical term 'genotype-by-environment interaction' or $G x E$ (Falconer 1960, 1989). $G x E$ variation comprises the extent to which genotypes differ in their patterns of phenotypic change over a given environmental range; it provides the raw material for plastic response patterns themselves to evolve (Via and Lande 1985; Scheiner 1993, 2006; Kruuk et al. 2008; Baythavong 2011; Chevin et al. 2013; see also Goldstein and Ehrenreich 2021 in this volume). As with any aspect of genetic diversity, populations and taxa differ in patterns of $G x E$ interaction variance as a result of previous selection, drift, and mutation (see references in Colautti et al. 2017).

Notice that as a result of $G x E$ variation-that is, of non-parallel norms of reaction-both the size and the rank order of trait differences among a given set of genotypes may vary from one environmental state to another (as is illustrated by the hypothetical genotypes shown in Figure 1.1). Genotypes may express different phenotypes in certain environments and converge on similar phenotypes in others; a genotype may have high or low fitness relative to others only in certain conditions (Falconer 1960, 1989; Gupta and Lewontin 1982; Conner and Hartl 2004; Scheiner 2006; Van Buskirk and Steiner 2009; Colautti et al. 2017). To the extent that fitnessrelated traits reflect this pervasive aspect of genetic diversity, the amount and pattern of genetic variation available to natural selection will depend on environmental context (Via and Lande 1987; Barton and Turelli 1989; Falconer and Mackay 1996). Such context-dependent or conditional expression of genetic differences can alter the pace and/or the direction of selective trajectories (Snell-Rood et al. 2010; van Dyken and Wade 2010; Lédon-Rettig et al. 2014). This insight has particular resonance with respect to the altered environments being rapidly created by human activities, since a novel environment can either trigger a selective event in a population by revealing genotypic differences, or buffer selection if genotypes express similar phenotypes in that environment (Sultan 2007).

The statistical quantity genotype-environment interaction reflects a biological fact: environmental conditions influence a genotype's expression, so phenotypic outcomes result from the particular developmental interaction of these two factors (see Lewontin 2000; West-Eberhard 2003; Mackay et al. 2009; Gilbert and Epel 2009, 2015). Although as biologists we are trained to seek single causes or 'main effects,' when the cause of a certain outcome is an interaction between two (or more) factors, the individual factors cannot be analytically separated, because the effect of one factor depends on the level of the other (Sokal and Rohlf 1987; Dodge 2003). In this case, the effect of having a given genotype on an individual's phenotype depends on its environmental state, just as the phenotypic effect on an individual of being in a given environmental state depends on its genotype (Griffiths 2006; Lewontin 2006).

This point has important implications for experimental analysis. In a conventional development experiment, several genotypes of interest (for instance a 'mutant' and a 'wild type') are compared in a single test environment. With this design, any phenotypic differences (other than developmental noise) result from the differences between the genotypes. If genotypes are instead compared in two or more test environments (as in a plasticity experiment), both environment and genotype as well as 
their interaction can be tested as potential sources of variation, using ANOVA or a variety of statistical approaches (Falconer 1960; Sokal and Rohlf 1987). Whenever norms of reaction are non-parallel (i.e., genotypes differ in the phenotypic change from one environmental treatment to another), the biological interaction that determines phenotypes will result in a statistical genotype-by-environment interaction term.

This changes how we analyze-and think about-genotype and environment as causal factors: in any dataset where the $G x E$ interaction term is significant, the 'main effects' of genotype and environment cannot be estimated as distinct factors (Lewontin 2006; Vitzthum 2003). As biostatisticians Sokal and Rohlf (1987, p. 198) explain, "many statisticians would not even test [the two main effects] once they found the interaction mean square to be significant, since in such a case an overall statement for each factor would have little meaning." In other words, it is largely meaningless to assess the causal impact of genotype alone on a trait when that impact depends on environmental state, and vice versa.

Accordingly, in the presence of $G x E$ variation, main effects that are tested offer only limited insight to trait variation. A main effect of genotype is most accurately interpreted simply as an indicator of an average effect across the study's environmental treatments, and likewise for a main effect of environment. Lack of a significant main effect does not mean that genotype or environment is unimportant. On the contrary, a significant $G x E$ term means they do contribute significantly to variation, but in an interactive rather than a fixed, additive way. This is demonstrated in Figure 1.2, where despite the dramatically different phenotypes produced by two genotypes in alternative environments, neither main effect would be statistically significant.

When $G x E$ is significant, just as it has "little meaning" to consider genotype and environment as separate phenotypic causes, estimating separate variance

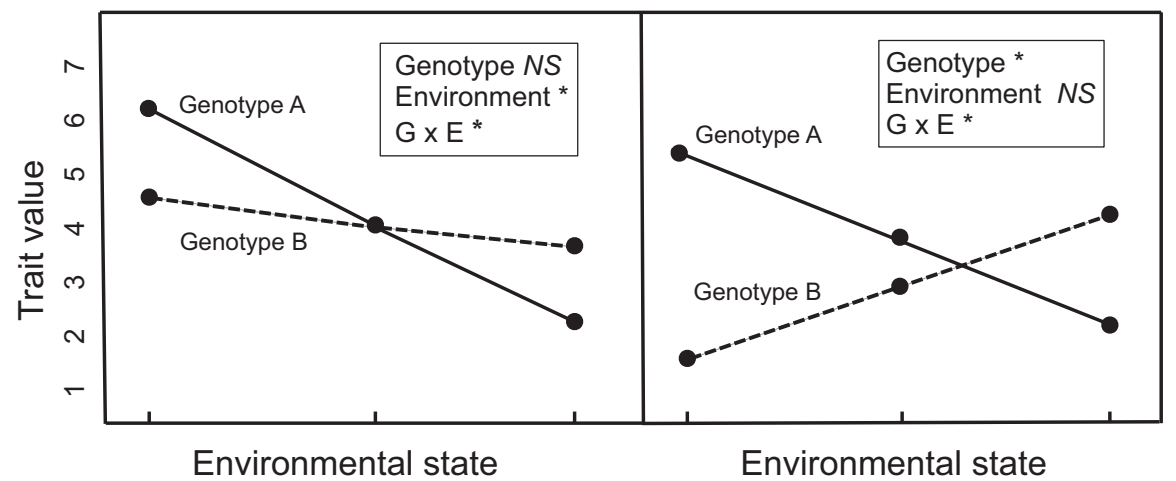

FIGURE 1.2 GxE interaction. Whenever norms of reaction are non-parallel (significant genotype-by-environment interaction term in ANOVA), genotype and environment cannot be meaningfully assessed as separate components of variance. This is illustrated here by two hypothetical datasets. On the left panel, there is no main effect of genotype even though the two genotypes clearly differ considerably; on the right, there is no main effect of environment despite a substantial impact of environmental state on trait value for each genotype. 
components for these terms is also questionable (Lewontin 2006). A pragmatic solution is to include the interaction variance component within the variance component for one of the main effect terms (Sokal and Rohlf 1987, Falconer 1989). This solution is conceptually problematic, however, since a researcher could equally justify including the interaction as part of the genotype effect, or as part of the environment effect! An alternative that avoids these interpretive problems is to assess the relative magnitudes of the main (i.e., average) effects of genotype and environment and of their interaction as descriptive statistics for a particular dataset rather than explanatory or causal ones, by simply comparing the effect sizes (for example, based on the mean square variance in an ANOVA; R.C. Lewontin, pers. comm).

Because genotype and environment jointly determine individual phenotypes, this developmental interaction shapes the variation expressed in natural populations. In other words, "it is on the product of genotype and environmental influences that natural selection acts" (Nager et al. 2000), so genetic diversity alone does not determine a population's evolutionary potential. Whenever naturally occurring genotypes express $G x E$ variation for relevant traits, fitness differences among genotypes-and hence the pace and outcome of selection-will depend on the distribution of environmental states in the field (Via and Lande 1985; Mitchell-Olds and Rutledge 1986; Scheiner 1993; Nager et al. 2000; Snell-Rood et al. 2010; van Dyken and Wade 2010; Lédon-Rettig et al. 2014; further references in Sultan 2015). For this reason, plasticity studies that characterize norms of reaction in response to field-based treatments (see next section) can most accurately test the evolutionary potential of real populations. Precise insight to $G x E$ results can be gained through post hoc tests, such as linear contrasts following ANOVA to determine whether genotypes differ significantly within a specific treatment of interest. This kind of detailed analysis can address targeted questions regarding a population's evolutionary potential, for instance under predicted future temperature or $\mathrm{CO}_{2}$ conditions (see Janes and Wayne 2006; Horgan-Kobelski et al. 2016) or when exposed to an introduced pathogen or other novel stressor.

\subsection{STUDYING PLASTICITY: ENVIRONMENTAL CUES AND PHENOTYPIC RESPONSES}

An organism's plastic expression-its norm of reaction-results from an underlying sequence of biological events. Depending on the organism's sensory and physiological systems, the individual perceives some aspect of the environment as a cue. This received signal is then transduced to initiate coordinated effects that lead to a characteristic phenotypic response, either by means of signaling molecules such as hormones and metabolites or by epigenetic modification (Dufty et al. 2002; Jaenisch and Bird 2003; Gottlieb 2004; Carroll et al. 2005; Cramer et al. 2011; Badeaux and Shi 2013; Lema and Kitano 2013; Morris and Mattick 2014; details and further references in Gilbert and Epel 2015; Sultan 2015). Such environmentally modulated regulatory pathways can directly alter physiology (for example by up-regulation of heat shock proteins; Queitsch et al. 2002) or can up- or down-regulate gene products including transcription factors and microRNA's so as to shape expression patterns of other genes and gene networks (Carroll et al. 2005; Cramer et al. 2011). In plasticity 
experiments, the researcher manipulates cues and documents responses of interest, whether those responses are morphogenetic or life-history outcomes, short-term behaviors or altered metabolic rates, changes to signaling pathways at the biochemical level, epigenomic modifications, or resulting transcriptome impacts.

Interdisciplinary approaches that integrate these layered modes of plasticity can provide important insights into systems of cue perception, transduction, and phenotypic response. In the cichlid fish Astatotilapia burtoni, for instance, different social encounters cue males to rapidly and reversibly switch between a brightly colored, aggressively territorial 'dominant' phenotype and a cryptic, nonaggressive, and nonreproductive 'subordinate' phenotype (Burmeister et al. 2005). Underlying these alternative life-history and behavioral phenotypes are neuroendocrine pathways that up- or down-regulate approximately 5\% of neural genes studied (Renn et al. 2008) and produce contrasting patterns of brain cell proliferation (Maruska et al. 2012).

A researcher's initial challenge is to identify and implement environmental treatments that will provide useful insight to the study organism's plastic responses. Here, the balance between experimental feasibility and realism is critical. The most informative design will recreate alternative conditions that elicit plastic responses in natural populations, yet do so with sufficient precision for results to be interpretable with respect to specific environmental cues (Miner et al. 2005; Groothuis and Taborsky 2015). This requires sufficient knowledge of the organism's biology and natural history to determine the environmental factors likely to be relevant and to choose naturalistic factor levels; plasticity studies that test easily manipulated but ecologically arbitrary treatments reveal little about the variability and potentially adaptive adjustments that might be expressed in real populations. Previous studies or pilot work with a given system can be invaluable in making these experimental choices.

Potential cues for an organism depend upon its evolved sensory and metabolic capacities-i.e., how it gathers information about its environment. In some cases, identifying a key cue for plastic response seems simple. For example, the amount of photosynthetically usable light is clearly a key component of the environment for plants. Yet more nuanced aspects of this seemingly straightforward environmental factor may substantially influence plant phenotypes, including the precise spectral distribution of available light (Smith 2000; Schlichting and Smith 2002; Franklin 2008; Ballaré 2009). With this awareness, a researcher might devise contrasting treatments that covary light intensity and spectral quality, for instance based on field measurements of alternative types of site or microsite that the species inhabits.

In other situations, the environmental cue that induces an organism's plastic response may be difficult to discern. The specific types of territorial interaction that cue the dramatic plastic responses of male A. burtoni cichlids described above provide a case in point. Complex cues have also been studied in several amphibian species known to express a crucial aspect of life-history plasticity. Tadpole larvae that occupy transient pools are well known to hasten their metamorphosis when these ponds begin to dry up, but it is not clear exactly how they perceive this impending change while they are still submerged. For one species of spadefoot toad (Scaphiopus hammondii), carefully designed lab studies were required to show that neither the higher water temperature nor the more frequent physical interactions with neighbors 
that result from diminishing water volume served as cues to initiate this timing change. Instead, the operative signal to speed metamorphosis consists of two perceived changes: the metabolic feedback effect of restricted foraging in the reduced volume of water, combined with the visual cue of greater proximity to the water's surface (Denver et al. 1998). Note that closely related species may have evolved to utilize distinct plasticity cues even for very similar adaptive responses. For instance, in $S$. couchii, a congeneric spadefoot toad species with similar metamorphic plasticity, larvae sense the imminent drying of ephemeral pools through more frequent physical contact with other tadpoles as the water volume decreases (Newman 1994). These cues (as well as indirect indicators of other potential risks such as predator presence) are perceived through the animal's sensory systems and then transduced via the amphibian neurohormonal stress pathway to shape developmental responses (Denver 2009, 2013).

Having identified a key environmental factor that cues plasticity (whether or not its transduction system is fully known), designing treatments also requires knowledge of its patterns of temporal and spatial variation in the field. Rather than vary the factor mean in alternative fixed treatments, sometimes it is more ecologically meaningful to vary the range, timing, duration, or periodicity of environmental states (Miner and Vonesh 2004). For instance, both the amount and the diurnal distribution of light vary in temperate forests as a result of canopy structure, though the latter is tricky to vary experimentally. In a meticulous field study, Wayne and Bazzaz (1993) showed that birch seedlings given the same total, reduced daily amount of photosynthetically active sunlight expressed very different plastic responses to this shade depending on whether the light was provided at a consistent, moderate level throughout the day or (as in a forest gap) in the form of very low light with a brief interval at full intensity. Treatments that vary temporal patterns for key factors can also show how quickly individual organisms can plastically adjust relevant functional traits, revealing timing differences of potential adaptive relevance among genotypes or taxa (e.g., Sultan et al. 1998; Bell and Sultan 1999). Experimental treatments may also be designed to covary an environmental factor of interest with another aspect of the organism's environment (Gebhardt and Stearns 1993). Research on plastic expression in predicted future environments may test an organism's response to combinations of $\mathrm{CO}_{2}$ concentration and temperature rather than to each factor alone (e.g., Miller et al. 2012). Such covariation designs can reveal important synergistic effects of factors that may co-occur. In general, field data on environmental variation will provide a robust basis for well-informed experimental design and for contextualizing the results (Miner et al. 2005).

As noted above, plastic responses may be studied at various levels of biological organization; a researcher's expertise and experimental goals will guide the choice of traits. An evolutionary, physiological, or behavioral ecologist might focus primarily on the expression of putatively adaptive plastic traits such as physiological adjustments, anatomical changes to tissues or organs, alternative life-histories or behaviors, and altered whole-body morphologies. As with environmental cues, knowledge of the organism's natural history is the essential guide to trait choice; environmental challenges known to vary temporally or spatially within populations of a given species can suggest candidate plasticity traits to measure. Plastic responses 
may be considered as functionally adaptive based on statistical associations with fitness (phenotypic selection analysis), but because environmental conditions affect both trait and fitness values these analyses can be strongly biased (Stinchcombe et al. 2002; see Scheiner et al. 2002 for a path-analytical approach). In cases where plastic trait expression is irreversible or can be experimentally manipulated, fitness benefits can be tested by direct comparing alternative outcomes within each environmental treatment (Schmitt et al. 2003). However, in many well-studied cases of plasticity, trait changes are interpreted as adaptive based simply on ecological or functional considerations; examples include predator- and herbivore-induced structural and chemical defenses; thermoregulatory morphological and behavioral phenotypes that minimize heat and cold stress; and allocational or structural changes to tissues that maximize a limiting resource, such as increased gill surface area of fish in hypoxic water, longer root systems of plants growing in nutrient or moisture-limited substrates, and enlarged or specialized feeding structures that maximize access to available foods (references in Gilbert and Epel 2015; Sultan 2015).

In contrast to studies focused at the level of trait expression, biologists who study genomic, cellular, endocrine, and neural systems might instead seek to determine underlying mechanisms of ecologically relevant trait plasticity by tracking signal transduction pathways, epigenetic marks, or transcription changes. In addition to elucidating plastic cue and response systems as such, mechanistic studies can provide new evolutionary insights by revealing unexpected convergences, shared signaling pathways, and similar epigenetic dynamics among diverse organisms as well as differences that indicate phylogenetic constraint or distinct selective histories (Niederhuth et al. 2016; Adrian-Kalchhauser et al. 2020). Indeed, plasticity is an exceptionally rich area for collaborative investigations that combine traditionally distinct approaches to link molecular mechanisms with ecological and evolutionary consequences.

\subsection{TRANSGENERATIONAL PLASTICITY AND MULTI-GENERATION NORMS OF REACTION: A MORE COMPLEX PICTURE}

An even more complex picture of phenotypic causation emerges when we consider how environmental conditions encountered during one generation may influence the next. Inherited positive and negative effects of maternal environment ('maternal effects') have long been familiar (in part through the work of plant and animal breeders) as a substantial non-genetic source of phenotypic variation (Falconer 1989). These effects may be transmitted to offspring via changes to the amount and composition of nutritive tissues packed by maternal individuals into seeds or eggs; these 'provisioning' changes often directly reflect resource availability to parent individuals and lead to congruent increases or decreases in offspring size or growth rate (Haig and Westoby, 1988; Fenner and Thompson, 2005; Hafer et al. 2011; Uller et al. 2013; Baker et al. 2018).

A more recent insight is that offspring phenotypes may be influenced by parental conditions in highly specific ways beyond provisioning effects. The precisely cued responses of individual organisms to their environments may extend beyond immediate adjustments to their own development, life-history, and behavior to 
include 'transgenerational plasticity'-trait-specific changes to offspring phenotypes induced by parental conditions (reviewed by Mousseau and Fox 1998; Agrawal et al. 1999; Uller 2008, 2013; Bonduriansky and Day 2009, 2018; Mousseau et al. 2009; Herman and Sultan 2011; Salinas et al. 2013; Donelson et al. 2018; see also Pfennig 2021 and Bonduriansky 2021 in this volume). Considerably more debate surrounds this aspect of plasticity, especially regarding its phenotypic impact beyond early life stages and hence its potential adaptive value and evolutionary role (Bossdorf et al. 2008; Badyaev and Uller 2009; Charlesworth et al. 2017; for theoretical investigations see references in Bonduriansky and Day 2018; Uller 2019). Fundamental questions also remain about the transmission mechanisms that underlie transgenerational plasticity, including whether heritable regulatory factors are environmentally induced or stochastic and how long such factors can persist across generations (Haig 2007; Jablonka and Raz 2009; Boyko and Kovalchuk 2011; Grossniklaus et al. 2013; van der Graaf et al. 2015). Below is a quick overview of this fascinating area of plasticity research (for further discussion, see Bonduriansky 2021 in this volume).

Adaptive transgenerational plasticity has been documented at the phenotypic level in a wide range of plant and animal taxa (e.g., Agrawal et al. 1999; Galloway and Etterson 2007; Herman et al. 2012; Miller et al. 2012; Shama and Wegner 2014). In these systems, parent individuals exposed to a particular abiotic or biotic stress altered offspring phenotypes in ways that specifically pre-adapted them to that stress. For instance, juveniles of the common reef fish Acanthochromis polyacanthus were able to acclimate to a stressful $3^{\circ} \mathrm{C}$ increase in water temperature (simulating future ocean conditions) if their parents had been exposed to elevated temperatures, due to heritable methylation changes in a number of oxygen-processing and metabolic genes (Ryu et al. 2018). Although in many cases the regulatory factors that mediate such adaptive transgenerational effects are not known, molecular studies point to several possible mechanisms. A specific parental environment may induce: (1) changes to heritably transmitted regulatory molecules such as hormones and small RNAs that are transferred into the embryo via maternal cytoplasm or sperm, and/ or (2) heritable maternal or paternal DNA or chromatin modifications that epigenetically 'mark' the offspring genome and alter its transcriptional activity (Soubry et al. 2014; Blake and Watson 2016; Quadrana and Colot 2016; Perez and Lehner 2019). Environmentally induced changes to DNA methylation state appear to be a particularly widespread mode of epigenetic change in plants and many animals that in some cases can persist for several descendent generations (Law and Jacobsen, 2010; He et al., 2011; Schubeler 2015; e.g., Dowen et al., 2012; Yu et al., 2013; Skinner, 2014; Colicchio et al. 2015; Baker et al. 2018). 'Adaptive transgenerational plasticity' is predicted to evolve when the parental environment reliably predicts the offspring environment, for instance in cases when offspring are likely to encounter the same specific stresses as the parent(s) (Agrawal et al., 1999; Galloway, 2005; Herman et al. 2014). Yet whether transgenerational effects induced by specific environmental stresses are a major source of adaptive variation across diverse natural systems remains to be robustly established (Uller et al. 2013).

What is already clear, however, is that environmental effects inherited from the previous generation (or possibly generations) can influence an organism's developmental response to its own environment: in other words, its norm of reaction (Miller 
et al. 2012; Plaistow et al. 2015; Sultan 2019). For example, herbivore damage to parental plants can 'prime' offspring to express faster or heightened responses if they are attacked, or can alter their threshold for response induction (Holeski et al. 2012 and references therein). In anemonefish, mean juvenile growth rate was sharply reduced in elevated dissolved $\mathrm{CO}_{2}$ compared with standard conditions, but when parents had been exposed to high $\mathrm{CO}_{2}$ concentrations their progeny developed normally in both environments, resulting in flatter norms of reaction across $\mathrm{CO}_{2}$ treatments (Miller et al. 2012). In mammals, parental stress or toxin exposure can lead to substantially different physiological and behavioral responses of juvenile and adult offspring to stresses they encounter (e.g. Crews et al. 2012; Gapp et al. 2014).

This point raises an important question about one of the main tenets of plasticity research. For over a century, the norm of reaction has been defined as a genotype's characteristic repertoire of responses to alternative conditions - "the expected phenotype of a given genotype as a function of the environment" (Chevin et al. 2010). This view guides both experimental design and evolutionary modeling. Yet if inherited environmental effects can alter the responses of a given genetic individual to its current conditions, the norm of reaction cannot be considered a fixed genotypic property. For example, seedling norms of reaction for Polygonum plant genotypes in response to sun and shade differ depending on whether their (maternal/paternal) parent had been grown in sun versus shade (Figure 1.3).

An alternative view is that the developing individual integrates environmental factors both past and present in genotype-specific ways, a perspective that suggests
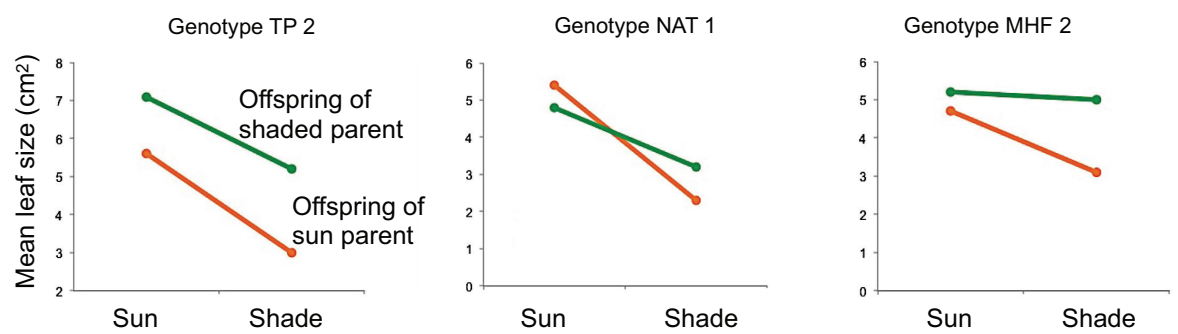

Offspring's developmental environment

FIGURE 1.3 Transgenerational effects on the norm of reaction. Environmental conditions experienced during the parental generation can alter developmental responses of offspring to their own environments, causing changes in the genotype's norm of reaction. This is illustrated here with data for three highly inbred genotypes of the annual plant Polygonum persicaria. Replicate parent individuals of each genotype were grown in greenhouse sun and shade treatments, and their progeny were grown in sun and shade. Offspring of each genotype expressed two different norms of reaction depending on their parent's environment. The parent environment effect on offspring reaction norm was genotype-specific: in TP2 (left), the progeny of shaded parents made larger leaves in both offspring environments, while in MHF 2 (right), the effect of parental shade was significant only for offspring developing in shade, and in NAT 1 (center), parental shade slightly reduced leaf size of offspring grown in sun. Two norms of reaction are shown for each genotype based on means of ten replicates from each parental environment in each progeny treatment. (Modified from Sultan 2017) 
several changes to research approaches (English et al. 2015; McNamara et al. 2016; Stein et al. 2018; Sultan 2019). Do transgenerational effects substantially alter norms of reaction, or can we continue to study plasticity as a genotypic property that is generally only slightly influenced by previous environments? Further studies testing multi-generation interactions between genotypes and environments in diverse biological systems are needed to resolve this central question about the causes of phenotypic variation.

\subsection{CONCLUSIONS}

The recognition that gene expression is environmentally sensitive has put an end once and for all to the misleading idea that genes and environment are alternative causes of phenotypic variation. Instead, it is now widely understood that plasticity-the variable expression of a given genotype in different environments-is an intrinsic property of organisms. As a result of both selective histories and evolved constraints, biological systems will differ characteristically with respect to the environmental signals that induce plastic responses; their transduction pathways; which traits are altered and precisely how; and the resulting context-dependent patterns of genetic variation. Box 1.1 provides some suggestions for future research. Investigating these dimensions of plasticity-either for a single taxon of interest or in a comparative framework-is key to understanding the phenotypic variation relevant to function and fitness, and hence to ecology and evolution.

\section{BOX 1.1 SUGGESTIONS FOR FUTURE RESEARCH}

- Identify the environmental factors and factor levels that act as plasticity cues for a given system. To predict variation patterns in natural populations, determine the distribution of the relevant environmental states within and among field sites or, in the case of transgenerational plasticity, from one generation to the next.

- Investigate precise pathways of environmental cue perception, transduction, trait expression changes, and (in the case of transgenerational effects) heritable transmission. This can be done through mechanistic studies of hormonal systems, metabolic feedbacks, or epigenetic modifications.

- Test the adaptive or maladaptive consequences of specific plastic responses in the context of environmental distributions, including predicted future conditions. This can clarify the short-term adaptive impact of both within-generation and transgenerational plasticity, a critical question with regard to rapid adaptive 'rescue' of populations under human-altered conditions.

- Assess future evolutionary potential by determining patterns of genotype-by-environment interaction variance for genotypes from natural populations, rather than quantifying allelic (sequence) variation per se. 
- Examine (both empirically and theoretically) how multi-generation $G x E$ interaction (i.e., environmental influences of two or more successive generations on fitness-related variation) may affect the process and strength of natural selection. Multi-generation studies may also provide exciting new ideas about how organisms integrate genetic factors, current environment, and previous environment to guide real-time phenotypic expression, and how these regulatory integration systems themselves evolve.

- For any of the above approaches, studies focusing on a species of interest can be valuably expanded by comparing populations, congeners or other close relatives, or different taxa from the same ecological community.

\section{REFERENCES}

Adrian-Kalchhauser, I., S. E. Sultan, L. Shama, H. Spence-Jones, S. Tiso, C. I. V. Keller, and F. J. Weissing. 2020. Understanding 'non-genetic' inheritance: Insights from molecular-evolutionary crosstalk. Trends Ecol. Evol. 35: 1078-1089.

Agrawal, A. A. 2001. Phenotypic plasticity in the interactions and evolution of species. Science 294:321-326.

Agrawal, A. A., C. Laforsch, and R. Tollrian. 1999. Transgenerational induction of defenses in animals and plants. Nature 401:60-63.

Atkinson, D., and M. Thorndyke. 2001. The nature of animal developmental ecology: Integrating the 'how' and the 'why', pp. 1-28. In D. Atkinson and M. Thorndyke, eds. Environment and Animal Development: Genes, Life Histories and Plasticity. BIOS Scientific Publishers, Oxford, UK.

Badeaux, A. and Y. Shi. 2013. Emerging roles for chromatin as a signal integration and storage platform. Nature Rev. Mol. Cell Biol. 14:211-224.

Badyaev, A. V. and T. Uller. 2009. Parental effects in ecology and evolution: mechanisms, processes and implications. Philos. Trans. R. Soc. Lond. B Biol Sci. 364:1169-77.

Baker, B. H., L. J. Berg, and S. E. Sultan. 2018. Context-dependent developmental effects of parental shade versus sun are mediated by DNA methylation. Front. Plant. Sci. 9: 1251

Ballaré, C. L. 2009. Illuminated behaviour: Phytochrome as a key regulator of light foraging and plant anti-herbivore defence. Plant Cell Env. 32:713-725.

Barton, N. H., and M. Turelli. 1989. Evolutionary quantitative genetics: How little do we know? Annu. Rev. Gen. 23:337-370.

Baythavong, B. S. 2011. Linking the spatial scale of environmental variation and the evolution of phenotypic plasticity: Selection favors adaptive plasticity in fine-grained environments. Am. Nat. 178:75-87.

Bell, D. L. and S. E. Sultan. 1999. Dynamic phenotypic plasticity for root growth in Polygonum: A comparative study. Am. J. Bot. 86:807-819.

Blake, G. E., and E. D. Watson. 2016. Unravelling the complex mechanisms of transgenerational epigenetic inheritance. Curr. Opin. Chem. Biol. 33:101-107.

Bonduriansky, R. 2021. Plasticity across generations. In D. W. Pfennig, ed., Phenotypic Plasticity and Evolution: Causes, Consequences, Controversies. CRC Press, Boca Raton, FL.

Bonduriansky, R., and T. Day. 2009. Nongenetic inheritance and its evolutionary implications. Annu. Rev. Ecol. Evol. Syst. 40: 103-125. 
Bonduriansky, R., and T. Day. 2018. Extended Heredity: A New Understanding of Inheritance and Evolution. Princeton University Press, Princeton, NJ.

Bonner, J. T. 1988. The Evolution of Complexity. Princeton University Press, Princeton, NJ.

Bossdorf, O., C. L. Richards, and M. Pigliucci. 2008. Epigenetics for ecologists. Ecol. Lett. 11:106-115.

Boyko, A., and I. Kovalchuk. 2011. Genome instability and epigenetic modificationheritable responses to environmental stress? Curr. Opin. Plant Biol. 14:260-266.

Brock, M. T., and C. Weinig. 2007. Plasticity and environment-specific covariances: An investigation of floral-vegetative and within flower correlations. Evolution 61:2913-2924.

Burmeister, S. S., E. D. Jarvis, and R. D. Fernald. 2005. Rapid behavioral and genomic responses to social opportunity. PLOS Biol. 3:e383.

Carroll, S. B., J. K. Grenier, and S. D. Weatherbee. 2005. From DNA to Diversity: Molecular Genetics and the Evolution of Animal Design (4th edition.). Blackwell Publishing, Malden, MA.

Charlesworth, D., N. H. Barton, and B. Charlesworth. 2017. The sources of adaptive variation. Proc. R. Soc. Lond. B Biol. Sci. 284:20162864.

Chevin, L.-M., R. Lande, and G. M. Mace. 2010. Adaptation, plasticity, and extinction in a changing environment: Towards a predictive theory. PLOS Biol. 8:e1000357.

Chevin, L.-M., S. Collins, and F. Lefèvre. 2013. Phenotypic plasticity and evolutionary demographic responses to climate change: taking theory out to the field. Funct. Ecol. 27:967-979.

Colautti, R. I., J. M. Alexander, K. M. Dlugosch, S. R. Keller, and S. E. Sultan. 2017. Invasions and extinctions through the looking glass of evolutionary ecology. Philos. Trans. R. Soc. Lond. B Biol Sci. 372:20160031.

Colicchio, J. M., F. Miura, J. K. Kelly, T. Ito, and L. C. Hileman. 2015. DNA methylation and gene expression in Mimulus guttatus. BMC Genomics 16:507.

Conner, J. K., and D. L. Hartl. 2004. A Primer of Ecological Genetics. Sinauer Associates, Sunderland, MA.

Coupe, S. A., B. G. Palmer, J. A. Lake, S. A. Overy, K. Oxborough, F.I. Woodward, J. E. Gray, and W. P. Quick. 2006. Systemic signaling of environmental cues in Arabidopsis leaves. J. Exp. Bot. 57:329-341.

Cramer, G. R., K. Urano, M. Pezzotti, and K. Shinozaki. 2011. Effects of abiotic stress on plants: A systems biology perspective. BioMed Centr. Plant Biol.11:163.

Crews, D., R. Gillette, S. V. Scarpino, M. Manikkam, M. I. Savenkova, and M. K. Skinner. 2012. Epigenetic transgenerational inheritance of altered stress responses. Proc. Natl. Acad. Sci. USA 109:9143-9148.

Denver, R. J., 2009. Stress hormones mediate environment-genotype interactions during amphibian development. Gen. Comp. Endocrinol. 164:20-31.

Denver, R. J., 2013. Neuroendocrinology of amphibian metamorphosis. Current topics in developmental biology, 103:195-227.

Denver, R. J., N. Mirhadi, and M. Phillips. 1998. Adaptive plasticity in amphibian metamorphosis: responses of Scaphiopus hammondii tadpoles to habitat desiccation. Ecology 79:1859-1873.

Des Marais, D. L., K. M. Hernandez, and T. E. Juenger. 2013. Genotype-by-environment interaction and plasticity: Exploring genomic responses of plants to the abiotic environment. Annu. Rev. Ecol. Evol. Syst. 44:5-29.

Dobzhansky, T. and B. Spassky. 1944. Genetics of natural populations. XI. Manifestation of genetic variants in Drosophila pseudoobscura in different environments. Genetics 29:270-290.

Dodge, Y. 2003. The Oxford Dictionary of Statistical Terms. Oxford University Press, Oxford, UK. 
Donelson, J. M., S. Salinas, P. L. Munday, and L. N. Shama. 2018. Transgenerational plasticity and climate change experiments: Where do we go from here? Glob. Ch. Biol. 24:13-34.

Dowen, R. H., M. Pelizzola, R. J. Schmitz, R. Lister, J. M. Dowen, J. R. Nery, J. E. Dixon, and J. R. Ecker. 2012. Widespread dynamic DNA methylation in response to biotic stress. Proc. Natl. Acad. Sci. USA 109:E2183-E2191.

Dufty Jr., A. M., J. Clobert, and A. P. Møller. 2002. Hormones, developmental plasticity and adaptation. Trends Ecol. Evol. 17:190-196.

English, S., I. Pen, N. Shea, and T. Uller. 2015. The information value of non-genetic inheritance in plants and animals. PLoS One 10:e0116996.

Falconer, D. S., 1960. Introduction to Quantitative Genetics. Oliver and Boyd, London.

Falconer, D. S. 1989. Introduction to Quantitative Genetics (3rd edition). Longman, London.

Falconer, D. S. and T. F. C. Mackay. 1996. Introduction to Quantitative Genetics (4th edition). Longman, Harlowe, London.

Fenner, M. and K. Thompson. 2005. The Ecology of Seeds. Cambridge University Press, Cambridge, UK.

Fischer, E. K., C. K. Ghalambor, and K. L. Hoke. 2016. Plasticity and evolution in correlated suites of traits. J. Evol. Biol. 29:991-1002.

Franklin, K. A. 2008. Shade avoidance. N. Phytol.179:930-944.

Galloway, L. F. 2005. Maternal effects provide phenotypic adaptation to local environmental conditions. N. Phytol. 166:93-100.

Galloway, L. F. and J. R. Etterson. 2007. Transgenerational plasticity is adaptive in the wild. Science 318:1134-1136.

Gapp, K., S. Soldado-Magraner, M. Alvarez-Sánchez, J. Bohacek, G. Vernaz, H. Shu, T. B. Franklin, D. Wolfer, and I. M. Mansuy. 2014. Early life stress in fathers improves behavioural flexibility in their offspring. Nature Comm. 5:1-8.

Gebhardt, M. D., and S. C. Stearns. 1993. Phenotypic plasticity for life history traits in Drosophila melanogaster. I. Effect on phenotypic and environmental correlations. J. Evol. Biol. 6:1-16.

Gilbert, S. F. 2001. Ecological developmental biology: developmental biology meets the real world. Dev. Biol. 233:1-12.

Gilbert, S. F. 2012. Ecological developmental biology: Environmental signals for normal animal development. Evol. Devel. 14:20-28.

Gilbert, S. F., and D. Epel. 2009. Ecological Developmental Biology: Integrating Epigenetics, Medicine, and Evolution. Sinauer Associates, Sunderland, MA.

Gilbert, S. F., and D. Epel. 2015. Ecological Developmental Biology: the Environmental Regulation of Development, Health, and Evolution (2nd edition). Sinauer Associates, Sunderland, MA.

Gillespie, J. H., and M. Turelli. 1989. Genotype-environment interactions and the maintenance of polygenic variation. Genetics 121:129-138.

Gilroy, S., and A. Trewavas. 2001. Signal processing and transduction in plant cells: The end of the beginning? Nat. Rev. Mol. Cell Biol. 2:307-314.

Goldstein, I. and I. M. Ehrenreich. 2021. Genetic variation in phenotypic plasticity. In D.W. Pfennig, ed., Phenotypic Plasticity and Evolution: Causes, Consequences, Controversies. CRC Press, Boca Raton, FL.

Gottlieb, G. 2004. Normally occurring environmental and behavioral influences on gene activity, pp. 85-106. In C. G. Coll, E. L. Bearer, and R. M. Lerner, eds., Nature and Nurture: The Complex Interplay of Genetic and Environmental Influences on Human Behavior and Development. Lawrence Erlbaum Associates, London.

Griffiths, P. E. 2006. Philip Kitcher, genetic determinism, and the informational gene, pp. 175-198. In E.M. Neumann-Held and C. Rehmann-Sutter, eds., Genes in Development: Re-reading the Molecular Paradigm. Duke University Press, Durham, NC. 
Groothuis, T., and B. Taborsky. 2015. Introducing biological realism into the study of developmental plasticity in behaviour. Front. Zool. 12:S6.

Grossniklaus, U., W. G. Kelly, A. C. Ferguson-Smith, M. Pembrey, and S. Lindquist. 2013. Transgenerational epigenetic inheritance: How important is it? Nat. Rev. Genet. 14:228-235.

Gupta, A. P., and R. C. Lewontin. 1982. A study of reaction norms in natural populations of Drosophila pseudoobscura. Evolution 36:934-948.

Hafer, N., S. Ebil, T. Uller, and N. Pike. 2011. Transgenerational effects of food availability on age at maturity and reproductive output in an asexual collembolan species. Biol. Lett. 7:755-758.

Haig, D. 2007. Weismann rules! Ok? Epigenetics and the Lamarckian temptation. Biol. Philos. 22:415-428.

Haig, D., and M. Westoby. 1988. Inclusive fitness, seed resources, and maternal care, pp. 60-79. In J. Lovett-Doust and L. L. Doust, eds., Plant Reproductive Ecology: Patterns and Strategies. Oxford University Press, Oxford, UK.

Haldane, J. B. S. 1946. The interaction of nature and nurture. Ann. Eugen. 13:197-205.

He, X. J., T. Chen, and J. K. Zhu. 2011. Regulation and function of DNA methylation in plants and animals. Cell Res. 21:442-465.

Herman, J. J. and S. E. Sultan. 2011. Adaptive transgenerational plasticity: Case studies, mechanisms, and implications for natural populations. Front. Plant Genet. Genom. 2:102.

Herman, J. J., S. E. Sultan, T. Horgan-Kobelski, and C. E. Riggs. 2012. Adaptive transgenerational plasticity in an annual plant: Grandparental and parental drought stress enhance performance of seedlings in dry soil. Integr. Comp. Biol. 52:1-12.

Herman, J. J., H. G. Spencer, K. Donohue, and S. E. Sultan. 2014. How stable 'should' epigenetic modifications be? Insights from adaptive plasticity and bet hedging. Evolution 68:632-643.

Hodge, A. 2009. Root decisions. Plant Cell Env. 32:628-640.

Holeski, L. M., G. Jander, and A. A. Agrawal. 2012. Transgenerational defense induction and epigenetic inheritance in plants. Trends Ecol. Evol. 27:618-626.

Horgan-Kobelski, T., S. Matesanz, and S. E. Sultan. 2016. Limits to future adaptation in the invasive plant Polygonum cespitosum: Expression of functional and fitness traits at elevated $\mathrm{CO}_{2}$. J. Hered. 107:42-50.

Huey, R. B., M. Carlson, L. Crozier, M. Frazier, H. Hamilton, C. Harley, A. Hoang, and J. G. Kingsolver. 2002. Plants versus animals: Do they deal with stress in different ways? Integr. Comp. Biol. 42:415-423.

Jablonka, E. and G. Raz. 2009. Transgenerational epigenetic inheritance: Prevalence, mechanisms, and implications for the study of heredity and evolution. Quart. Rev. Biol. 84:131-176

Jaenisch, R., and A. Bird. 2003. Epigenetic regulation of gene expression: How the genome integrates intrinsic and environmental signals. Nat. Genet. 33:245-254.

Janes, D. E., and M. L. Wayne. 2006. Evidence for a genotype x environment interaction in sex-determining response to incubation temperature in the leopard gecko. Eublepharis Macularius Herpetologica 62:56-62.

Keller, E. F., and D. Harel. 2007. Beyond the gene. PLoS One 2: e1231.

Kruuk, L. E. B., J. Slate, and A.J. Wilson. 2008. New answers for old questions: The evolutionary quantitative genetics of wild animal populations. Annu. Rev. Ecol. Evol. Syst. 39:525-548.

Law, J. A., and S. E. Jacobsen. 2010. Establishing, maintaining and modifying DNA methylation patterns in plants and animals. Nat. Rev. Genet. 11:204-220.

Ledón-Rettig, C. C., D. W. Pfennig, A. J. Chunco, and I. Dworkin. 2014. Cryptic genetic variation in natural populations: A predictive framework. Integr. Comp. Biol. 54:783-793. 
Lema, S. C., and J. Kitano. 2013. Hormones and phenotypic plasticity: Implications for the evolution of integrated adaptive phenotypes. Curr. Zool. 59:506-525.

Lemos, B., C. R. Landry, P. Fontanillas, S. C. P. Renn, R. Kulathinal, K.M. Brown, and D. Hartl. 2008. Evolution of genomic expression, pp. 81-118. In M. Pagel and A. Pomiankowski, eds., Evolutionary Genomics and Proteomics. Sinauer Associates, Sunderland, MA.

Lewontin, R. C. 2000. The Triple Helix: Gene, Organism and Environment. Harvard University Press, Cambridge MA.

Lewontin, R. C. 2006 The analysis of variance and the analysis of causes (reprinted from J. Hum. Genetics 1974). Intl. J. Epidemiol. 35:520-525.

Mackay, T. F., E. A. Stone, and J. F. Ayroles. 2009. The genetics of quantitative traits: Challenges and prospects. Nat. Rev. Genet. 10:565-577.

Maruska, K. P., R. E. Carpenter, and R.D. Fernald. 2012. Characterization of cell proliferation throughout the brain of the African cichlid fish Astatotilapia burtoni and its regulation by social status. J. Comp. Neurol. 520:3471-3491.

McNamara, J. M., S. R. X. Dall, P. Hammerstein, and O. Leimar. 2016. Detection vs. selection: Integration of genetic, epigenetic and environmental cues in fluctuating environments. Ecol. Lett. 19:1267-1276.

Miller, G. M., S-A. Watson, J. M. Donelson, M. I. McCormick, and P. L. Munday. 2012. Parental environment mediates impacts of increased carbon dioxide on a coral reef fish. Nat. Clim. Ch. $2: 858-861$.

Miner, B. G., and J. R. Vonesh. 2004. Effects of fine grain environmental variability on morphological plasticity. Ecol. Lett. 7:794-801.

Miner, B. G., S. E. Sultan, S. G. Morgan, D. Padilla, and R. A. Relyea. 2005. Ecological consequences of phenotypic plasticity. Trends Ecol. Evol. 20:685-692.

Mitchell-Olds, T., and J. J. Rutledge. 1986. Quantitative genetics in natural plant populations: A review of the theory. Am. Nat. 127:379-402.

Moczek, A. P., S. E. Sultan, S. Foster, C. Ledón-Rettig, I. Dworkin, H. F. Nijhout, E. Abouheif and D. W. Pfennig. 2011. The role of developmental plasticity in evolutionary innovation. Proc. R. Soc. Lond. B Biol. Sci. 278:2705-2713.

Morris, K. V. and J. S. Mattick. 2014. The rise of regulatory RNA. Nat. Rev. Gen. 15:423-437.

Mousseau, T. A., and C. W. Fox. 1998. The adaptive significance of maternal effects. Trends Ecol. Evol. 13:403-407.

Mousseau, T. A., T. Uller, E. Wapstra, and A. V. Badyaev. 2009. Evolution of maternal effects: Past and present. Philos. Trans. R. Soc. Lond. B Biol Sci. R. Soc. (B) 364:1035-1038.

Nager, R. G., L. F. Keller, and A. J. Van Noordwijk. 2000. Understanding natural selection on traits that are influenced by environmental conditions, pp. 95-115. In T. Mousseau, B. Sinervo, J. A. Endler, eds., Adaptive Genetic Variation in the Wild. Oxford University Press, New York.

Newman, R. A. 1994. Effects of changing density and food level on metamorphosis of a desert amphibian, Scaphiopus couchii. Ecology 75:1085-1096.

Niederhuth, C. E., Bewick, A. J., Ji, L., Alabady, M. S., Do Kim, K., Li, Q., Rohr, N. A., Rambani, A., Burke, J. M., Udall, J. A. and Egesi, C. 2016. Widespread natural variation of DNA methylation within angiosperms. Genome Biol. 17:1-19.

Nijhout, H. F. 2003. Development and evolution of adaptive polyphenisms. Evol. Devel. 5:9-18.

Perez, M. F. and B. Lehner. 2019. Intergenerational and transgenerational epigenetic inheritance in animals. Nature Cell Biol. 21:143-151.

Pfennig, D. W. 2021. Key questions about phenotypic plasticity. In D. W. Pfennig, ed., Phenotypic Plasticity and Evolution: Causes, Consequences, Controversies. CRC Press, Boca Raton, FL.

Piersma, T. and Drent, J., 2003. Phenotypic flexibility and the evolution of organismal design. Trends Ecol. Evol. 18:228-233. 
Piersma, T. H. and Å. Lindström. 1997. Rapid reversible changes in organ size as a component of adaptive behaviour. Trends Ecol. Evol. 12:134-138.

Plaistow, S. J., C. Shirley, H. Collin, S. J. Cornell, and E.D. Harney. 2015. Offspring provisioning explains clone-specific maternal age effects on life history and life span in the water flea, Daphnia Pulex. Am. Nat. 186:376-389.

Portin, P., and A. Wilkins. 2017. The evolving definition of the term "gene". Genetics 205:1353-1364.

Quadrana, L., and V. Colot. 2016. Plant transgenerational epigenetics. Ann. Rev. Gen. 50:467-491.

Queitsch, C., T. Sanster, and S. Lindquist. 2002. Hsp90 as a capacitor of phenotypic variation. Nature 417:618-624.

Renn, S. C., N. Aubin-Horth, and H. A. Hofmann. 2008. Fish and chips: Functional genomics of social plasticity in an African cichlid fish. J. Exp. Biol. 211:3041-3056.

Rivera-Yoshida, N., A. V. Arzola, J. A. Arias Del Angel, A. Franci, M. Travisano, A. E. Escalante and M. Benítez. 2019. Plastic multicellular development of Myxococcus xanthus: Genotype-environment interactions in a physical gradient. R. Soc. Open Sci. 6:181730.

Ryu, T., H. D. Veilleux, J. M. Donelson, P. L. Munday, and T. Ravasi. 2018. The epigenetic landscape of transgenerational acclimation to ocean warming. Nat. Clim. Ch. 8:504-509.

Salinas, S., S. C. Brown, M. Mangel, and S. B. Munch. 2013. Non-genetic inheritance and changing environments. Non-Gen. Inher. 1:38-50.

Sarkar, S., 2004. From the Reaktionsnorm to the evolution of adaptive plasticity, pp. 10-30. In S. M. Scheiner, T. J. DeWitt, eds., Phenotypic Plasticity: Functional and Conceptual Approaches. Oxford University Press, New York.

Scheiner, S. M. 1993. Genetics and evolution of phenotypic plasticity. Annu. Rev. Ecol. Syst. 24:35-68.

Scheiner, S. M. 2006. Genotype-environment interactions and evolution, pp. 326-338. In C. W. Fox and C. M. Wolf, eds., Evolutionary Genetics: Concepts and Case Studies. Oxford University Press, London.

Scheiner, S. M., K. Donohue, L. A. Dorn, S. J. Mazer, and L. M. Wolfe. 2002. Reducing environmental bias when measuring natural selection. Evolution 56:2156-2167.

Schlichting, C. D., and H. Smith. 2002. Phenotypic plasticity: Linking molecular mechanisms with evolutionary outcomes. Evol. Ecol. 16:189-211.

Schmitt J., J. R. Stinchcombe, M. S. Heschel, and H. Huber. 2003. The adaptive evolution of plasticity: Phytochrome-mediated shade avoidance responses. Integr. Comp. Biol. 43:459-469.

Schubeler, D . 2015. Function and information content of DNA methylation. Nature 517:321-326.

Seymour, J. R., R. Simó, T. Ahmed, and R. Stocker. 2010. Chemoattraction to dimethylsulfoniopropionate throughout the marine microbial food web. Science 329:342-345.

Shama, L. N., and K. M. Wegner. 2014. Grandparental effects in marine sticklebacks: Transgenerational plasticity across multiple generations. J. Evol. Biol. 27:2297-2307.

Skinner, M. K. 2014. Endocrine disruptor induction of epigenetic transgenerational inheritance of disease. Mol. Cell. Endocrin. 398:4-12.

Smith, H., 2000. Phytochromes and light signal perception by plants-an emerging synthesis. Nature 407:585-591.

Snell-Rood, E. C., J. D. Van Dyken, T. Cruickshank, M. J. Wade, and A. P. Moczek. 2010. Toward a population genetic framework of developmental evolution: The costs, limits, and consequences of phenotypic plasticity. BioEssays 32:71-81.

Sokal, R. R., and F. J. Rohlf. 1987. Introduction to Biostatistics (2nd edition). Dover Publications, Mineola, NY.

Soubry, A., C. Hoyo, R. L. Jirtle, and S. K. Murphy. 2014. A paternal environmental legacy: Evidence for epigenetic inheritance through the male germ line. BioEssays 36:359-371. 
Stearns, S. C. 1989. The evolutionary significance of phenotypic plasticity. Bioscience 39:436-445.

Stebbins, G. L. 1980. Botany and the synthetic theory of evolution, pp. 139-152. In E. Mayr and W. B. Provine, eds. The Evolutionary Synthesis. Harvard University Press, Cambridge, MA.

Stein, L. R., S. A. Bukhari, and A. M. Bell. 2018. Personal and transgenerational cues are nonadditive at the phenotypic and molecular level. Nat. Ecol. Evol. 2:1306-1311.

Stinchcombe, J. R., M. T. Rutter, D. S. Burdick, P. Tiffin, M. D. Rausher and R. Mauricio. 2002. Testing for environmentally induced bias in phenotypic estimates of natural selection: Theory and practice. Am. Nat. 160:11-523.

Stinchcombe, J. R., Function-valued Trait Working Group, and M. Kirkpatrick. 2012. Genetics and evolution of function-valued traits: understanding environmentally responsive phenotypes. Trends Ecol. Evol. 27:637-647.

Sultan, S. E. 1992. Phenotypic plasticity and the Neo-Darwinian legacy. Evol. Trends Plants 6:61-71.

Sultan, S. E. 2000. Phenotypic plasticity for plant development, function and life-history. Trends Plant Sci. 5:537-542.

Sultan, S. E. 2007. Development in context: The timely emergence of eco-devo. Trends Ecol. Evol. 22:575-582.

Sultan, S. E. 2015. Organism and Environment: Ecological Development, Niche Construction, and Adaptation. Oxford University Press, London and New York.

Sultan, S. E. 2017. Developmental plasticity: re-conceiving the genotype. Royal Society Interface Focus 7: 20170009.

Sultan, S. E. 2019. Genotype-environment interaction and the unscripted reaction norm, pp. 109-126. In T. Uller and K. Laland, eds., Cause and Process in Evolution. Vienna Series in Theoretical Biology, MIT Press, Cambrdge, MA.

Sultan, S. E., and S. C. Stearns. 2005. Environmentally contingent variation: Phenotypic plasticity and norms of reaction, pp. 303-332. In B. Hall and B. Hallgrimsson, eds., Variation: A Hierarchical Examination of a Central Concept in Biology. Elsevier Academic Press, New York.

Sultan, S. E., A. M. Wilczek, D. L. Bell, and G. Hand. 1998. Physiological response to complex environments in annual Polygonum species of contrasting ecological breadth. Oecologia 115:564-578.

Trewavas, A. J. 2015. Plant Behaviour and Intelligence. Oxford University Press, London.

Uller, T. 2008. Developmental plasticity and the evolution of parental effects. Trends Ecol. Evol. 23:432-438.

Uller T. 2013. Non-genetic inheritance and evolution, pp. 267-287. In K. Kampourakis ed., The Philosophy of Biology: History, Philosophy and Theory of the Life Sciences, vol. 1. Springer, Dordrecht, Germany.

Uller, T. 2019. Evolutionary perspectives on transgenerational epigenetics. Transgenerational Epigenetics (2nd ed.). Transl. Epigenetics 13:333-350.

Uller, T., S. Nakagawa, and S. English. 2013. Weak evidence for anticipatory parental effects in plants and animals. J. Evol. Biol. 26:2161-2170.

Van Buskirk, J., and U. Steiner. 2009. The fitness costs of developmental canalization and plasticity. J. Evol. Biol. 22:852-860.

van den Hout, P. J., T. Piersma, A. Dekinga, S. K. Lubbe, and G. H. Visser. 2006. Ruddy turnstones Arenaria interpres rapidly build pectoral muscle after raptor scares. J. Avian Biol. 37:425-430.

Van Der Graaf, A., R. Wardenaar, D. A. Neumann, A. Taudt, R. G. Shaw, R. C. Jansen, R. J. Schmitz, M. Colomé-Tatché, and F. Johannes. 2015. Rate, spectrum, and evolutionary dynamics of spontaneous epimutations. Proc. Natl. Acad. Sci. USA. 112:6676-6681.

Van Dyken, J. D. and M. J. Wade. 2010. The genetic signature of conditional expression. Genetics 184:557-570. 
Via, S., and R. Lande. 1985. Genotype-environment interaction and the evolution of phenotypic plasticity. Evolution 39:505-522.

Via, S., and R. Lande. 1987. Evolution of genetic variability in a spatially heterogeneous environment: effects of genotype-environment interaction. Genet. Res. 49:147-156.

Vitzthum, V. J. 2003. A number no greater than the sum of its parts: The use and abuse of heritability. Hum. Biol. 1:539-558.

Walbot, V. 1996. Sources and consequences of phenotypic and genotypic plasticity in flowering plants. Trends Plant Sci. 1:27-32.

Wayne, P., and F. A. Bazzaz. 1993. Birch seedling responses to daily time courses of light in experimental forest gaps and shadehouses. Ecology 74:1500-1515.

West-Eberhard, M. J. 2003. Developmental Plasticity and Evolution. Oxford University Press, New York.

Wray, G. A., H. E. Hoekstra, D.J. Futuyma, R.E. Lenski, T.F.C. Mackay, D. Schluter, and J. E. Strassman. 2014. Does evolutionary theory need a rethink? No, all is well. Nature 514:161-164.

Yu, A., G. Lepère, F. Jay, J. Wang, L. Bapaume, Y. Wang, A.-L. Abraham, J. Penterman, R. L. Fischer, and O. Voinnet. 2013. Dynamics and biological relevance of DNA demethylation in Arabidopsis antibacterial defense. Proc. Natl. Acad. Sci. USA. 110: 2389-2394. 